\title{
Monoclonal antibody and blood plasma ABO blood group based therapy against COVID-19
}

\author{
Kalayu Shimuye ${ }^{1}$, Jianda $\mathrm{Hu}^{1}$, and Minhui $\operatorname{Lin}^{1}$ \\ ${ }^{1}$ Fujian Medical University
}

May 29, 2020

\begin{abstract}
In new decade new coronavirus emerged. By April 2020, there is no accepted antiviral drug or antibody against COVID-19. COVID-19 (SARS-CoV-2) has nucleic acid sequence similarity $96 \%$ with bat coronavirus, $79.6 \%$ SARS-CoV-1. SARS-CoV2 and SARS-CoV-1have common human host-cell ACE2 receptor. This similarity helps for effective vaccine and antibody development. At Wuhan, China, convalescent plasma therapy achieved $70 \%$ recovery results. ABO blood group susceptibility study revealed O blood group were very low risk whereas A were at high risk against COVID-19. ABO natural antibodies have positive effect to slowdown COVID-19 in less hygienic environment (less developed region). Isolation of specific antibody from EBV transformed B-lymphocyte recovered patients is encouraged. Urgent production of potent neutralizing antibody and vaccine is required and we identified sensitive immunogenic amino acid segment (318-510) in S1-protein domain that contains important and essential amino acids including cysteine, glutamic acid and aspartic acid, which associated with ACE2 expression.
\end{abstract}

\section{Introduction}

In the end of 2019, early of 2020 unknown pneumonia caused very contagious pathogen was reported at Wuhan City, Hubei province, China. Novel corona virus in respiratory specimens was detected by next generation sequencing or real-time RT-PCR methods. ${ }^{1}$ Chinese scientist released the complete sequenced of the novel corona virus (WH-Human_1) on January 10, 2020 and it is freely online available in the Gene Bank. ${ }^{2}$ (ICTV) International Committee on Taxonomy of Viruses named the virus severe acute respiratory syndrome corona virus 2 (SARS-CoV-2). Later on, the World Health Organization (WHO) was official named the novel coronavirus as COVID-19. There are four type of corona virus: $\alpha$-coronavirus $(\alpha-\mathrm{COV}), \beta$-coronavirus $(\beta-$ $\mathrm{COV}), \delta$-coronavirus ( $\delta$-COV) and $\gamma$ - coronavirus $(\gamma-\mathrm{COV})$. This novel coronavirus (COVID-19) is belong to $\beta$-coronavirus, which is the most cosmopolitan virus in nature. Coronavirus is an RNA virus with single strand and about 80-120 $\mathrm{nm}$ in diameter. ${ }^{3}$ Before the end of 2019, there were 6 known coronavirus infect human and cause respiratory diseases. Of those six corona virus 229E, OC43, NL63,HKU, SARS-CoV-1 and MERS-CoV; SARS-Co-V-2 (COVID-19) became the seventh human corona virus and globally pandemic in $2020 .{ }^{4}$ The genomic sequence analysis of the novel corona virus revealed that the genomic sequence similarity shared with bat SARS coronavirus (SARSr-CoV-RaTG13) $96 \%{ }^{5}$ and SARS-CoV-1 (about 79\%) and MERS$\mathrm{CoV}$ (about 50\%). Bat may be an important natural reservoir of the virus. Moreover, Phylogenetic analysis and homology modelling revealed that COVID-19 had a similar receptor-binding domain structure to that of SARS-CoV-1, despite amino acid variation at some key residues. ${ }^{6,7}$ The infectivity and transmission rate of COVID-19 is higher than that of SARS and MRES coronavirus indeed its mortality rate is unknown yet. ${ }^{8}$

Human beings are confronting a pandemic in COVID-19, due to the reason that the virus is new human pathogen so far there are no licensed vaccines or antibodies. Convalescence serum based therapy from fully recovered COVID-19 patient is one of the best option for immediate therapy. In China, at Wuhan the convalescent serum able to recovered 70\% against COVID-19 patients. A scientific study from the previous outbreak of SARS-COV-1 enzyme-linked immunosorbent assay (ELISA) and Western blot neutralization assay 
results confirmed that the anti-SARS antibodies taken from the convalescent serum enabled neutralized the virus. ${ }^{9}$ The genomic sequence result indicated that this new virus (SARS-COV-2) shared the same receptors with the pervious SARS-COV-1) virus. ${ }^{6}$ Thus identify the receptor cells and produce monoclonal antibody against COVID-19 is important. In recent years, technology of monoclonal antibody become a powerful tool in diagnostic and therapeutic of various human Cancer and virus including Nipah, Ebola, Chikungunya, Zika, Middle East respiratory syndrome coronavirus (MERS-CoV), Severe Acute respiratory syndrome coronavirus (SARS-CoV-1) and more recently novel coronavirus or SARS-CoV-2). ${ }^{10}{ }^{11}$ The relationship between the ABO Blood group and the COVID-19 susceptibility was reported. ${ }^{12}$ This has relationship with the antibody found in plasma, which indicated that the natural antibody found in the blood plasma could inhibit the interaction of the virus to its host receptor cell entry gate. ${ }^{13}$ In this article, we suggests that convalescent serum from fully recovered COVID-19 patients as an immediate therapy can aligned with ABO blood group COVID-19 non-susceptible ( lower risk) individuals. Most importantly, identify the immunogenic parts of the new pathogen (COVID-19) would lead to produce effective monoclonal antibody and vaccine.

\section{Methods}

A literature search of Monoclonal antibody and blood plasma ABO blood group based therapy against COVID-19 was carried out for English language review articles, using the electronic database of PubMed and Google Scholars. The following search terms were input: (Monoclonal antibody, COVID-19, Spiked protein, ACE2, ABO blood group) in (Title/Abstract). The authors investigated all relevant studies for quality and publication date and notably, a list of references was added manually.

\section{COVID-19 as global pandemic}

In the new decade, new coronavirus become a big challenge to our world. In 21 first century COVID-19 come to be one the most contagious and world threat coronavirus pathogens. In the last month of 2019, COVID-19 case was reported from China, Wuhan. Wuhan is the most populous city in central China with a population exceeding 11 million. ${ }^{14}$ Later on, COVID-19 confirmed cases increased more than 10 times cases as Severe Acute Respiratory Syndrome Coronavirus (SARSCoV-1) and Middle East respiratory syndrome (MERS). COVID-19 moved very fast from endemic to epidemic finally World Health Organization (WHO) declared march 11 , 2020 COVID-19 as pandemic to the world. ${ }^{15}$ On April 9, 2020, the COVID-19 pandemic cases distributed to over 215 regions (Countries). Globally, so far millions were COVID-19 positive confirmed cases. It is increasing very fast every day and hundred thousands of lives are losing. In China, Wuhan where the epi-center of the COVID-19 was lockdown for about 76 days. In China, the COVID-19 cases showed decreasing significantly, with the high recovery rate although there is no licensed antiviral drug or vaccine consistently applied. This time it is necessary the entire world work together and defeat COVID-19 pandemic. Since March 2020, the COVID-19 is increased in alarming rates in large number of cases at Europeans and North Americans. This time, low-income countries mainly African and South Asian need to get ready and save lives by slow down the circulation of the virus. To Combat COVID-19 drastic action should be taken, there is no time to waste.

Figure 1. COVID-19 Pandemic globally by April 2020, from World Health Organization (WHO) report.

\section{Convalescent plasma as possible therapy against COVID-19}

Emil von Behring discovered serum therapy. In 1901, he received the noble prize award for his work use in the treatment serum diphtheria antitoxin. ${ }^{16}$ By that time, he was widely known as a savior of children, because as diphtheria was the major cause of child death. Scientific studies indicated that SARS-CoV-2 (COVID-19) has a genome size of $\sim 30$ kilobases, like other coronaviruses, encodes for multiple structural and non-structural proteins. The structural proteins include the spike (S) protein, the envelope (E) protein, the membrane $(\mathrm{M})$ protein, and the nucleocapsid $(\mathrm{N})$ protein. A set of $\mathrm{B}$ cell and $\mathrm{T}$ cell epitopes derived from the spike (S) and nucleocapsid (N) proteins were identified this might be helpful for effective antibody production and vaccine design against COVID-19. ${ }^{17}$ SARS-CoV-2 and SARS-CoV-1 shared a common human host-cell receptor protein, angiotensin converting enzyme 2 (ACE2) ${ }^{18}$ In 2009, intensive care unit recruited H1N1 
influenza virus infected patients shown that after treatment with convalescent plasma reduced respiratory tract viral load, serum cytokine response, and mortality. ${ }^{19}$ The convalescent serum plasma therapy experience of its efficacy and safety usage was reported from previously outbake of Middle East respiratory syndrome coronavirus (MERS-CoV). ${ }^{20}$ The convalescent plasma therapy has been used for the treatment SARS-CoV-1 patients in 2003 at Hong Kong which reported as good outcome. ${ }^{21}$

In Taiwan, during 2003 SARS-CoV-1 outbreak, the convalescent plasma taken from O blood type fully recovered patients for the infected healthcare worker resulted in zero viral load after $24 \mathrm{~h}$ of transfusion. Moreover, after convalescent plasma therapy one infected pregnant healthcare worker deliver healthy newborn baby with positive anti-SARS-CoV1 IgG. Indicated that the possibility of passive immunity transfer of antiSARS-CoV-1 antibody from the mother to the newborn. ${ }^{22}$

Recently, it has been reported that discharged patients in China and elsewhere were 23 testing positive after recovering. The situation was as unclear. Animal model based study was carrier out to reveals the reinfection and relapse of COVID-19. After treatment with convalescent plasma the pre-infected monkey shown neither viral loads in nasopharyngeal and anal swabs along timeline nor viral replication in all primary tissue compartments at 5 days post-reinfection. ${ }^{23}$ Generally, the viremia peaks most probably is high in the first week of the viral infection. Therefore, providing the convalescent plasma as early as the viral infection would make it more effective. ${ }^{24,25}$

At China where the epicenter of COVID-19, at currently in 2020 Convalescent plasma therapy has been used as a potential therapy for SARS-CoV-2 infected sever patients. In chia this able to cure 7 patients out of 10 patients. The procedure, important, profession and all technical details experiences shared from China, are in the reference.$^{24}$

ABO blood group antibody Inhibition the SARS Corona virus interaction with ACE2 host receptors cells as a potential for treatment and vaccine development against COVID-19

Susceptibility of peoples by SARS-CoV-2 (COVID-19) has influenced by various factors. In recently, age and gender was considered as a risk factor for susceptibility of COVID-19. Elderly peoples, male and patients with pre-exiting severe diseases are reported as vulnerable group by COVID-19. ${ }^{1,26}$ ABO blood group genetic factors shown significant effect on the peoples susceptibility by COVID-19. ${ }^{12}$ From the previous study, the association of ABO blood group and susceptibility to the Hepatitis B Hepatitis C, HIV and SARS-CoV-1 viruses were reported. ${ }^{27,28}$ The ABO blood types that discovered by Karl Landsteiner are determined based on the carbohydrate epitopes which presents on the surface of the human erythrocytes. ${ }^{12}$ Studies reported form USA and in recently from China, Wuhan from the 4 main blood groups $\mathrm{A}, \mathrm{B}, \mathrm{AB}$ and $\mathrm{O}$, group $\mathrm{O}$ is the most common one. ${ }^{12,29}$ In 2003 SARS CoV-1 outbreak, the O blood group individuals were shown to have very low infections as compared to non-O individuals. This is due the $\mathrm{ABO}$ gene effect on the virus protection. ${ }^{28}$ Surprisingly in the same fashion, the recent study in China, Wuhan revealed that the People with blood group A have a significantly higher risk for acquiring COVID-19 compared with non-A blood groups, whereas blood group $\mathrm{O}$ has a significantly lower risk for the infection compared with non-O blood groups. ${ }^{12}$ The major reason was due to the secret potential natural antibody found in the blood group $\mathrm{O}$ individuals could block the virus spiked protein interaction with the host angiotensin-converting enzyme at the entry gate. ${ }^{13}$ Moreover, blood group O has shown less risk of infection with Hepatitis B and HIV virus whereas group A are more prone to get Hepatitis B and HIV virus. This might be the blood group O has the natural immune potential in in protecting against blood-transmitted infection. Further genetic based detail studies are needed. ${ }^{27}$

Table 1 . ABO blood group antigen in blood cells and antibody in plasma compatibility

\begin{tabular}{lllll}
\hline Patient blood Type & Antigen in Red Blood Cells & Antibody in Plasma & Can receive from Red Blood Cells types & Can r \\
\hline A & A & Anti-B & A,O & A,AB \\
B & B & Anti-A & B,O & B,AB \\
AB & AB & None & O & O,A,I
\end{tabular}




\begin{tabular}{lllll}
\hline Patient blood Type & Antigen in Red Blood Cells & Antibody in Plasma & Can receive from Red Blood Cells types & Can r \\
\hline $\mathrm{O}$ & None & Anti-A and Anti-B & A, B, AB, O & AB \\
RhD positive & & RhD negative, RhD positive & RhD \\
RhD negative & & RhD negative & RhD \\
\hline
\end{tabular}

From the structural protein of coronavirus the spiked glycoprotein play very essential role in the entry of the corona virus in to the host cells. The name of coronavirus also related by its S-protein have a crown appearance which is derived their name; corona in Latin means crowns. The spiked protein has the receptorbinding domain (RBD). Thus, the virus use S-protein to bind its receptor with host cells by its S1 subunit followed by fused the viral and hast cell membrane through its S2 subunits. ${ }^{30,31}$ S-proteins coronavirus are the large heavily $\mathrm{N}$-glycosylated proteins which mediate the interaction of the virus with the host cell surface receptors. ${ }^{30,32}$ Angiotensin-converting enzyme 2 (ACE2) is an entry receptor on human for SARS-CoV2 and SARS-CoV-1 13,18,33-35 Angiotensin-converting enzyme 2 (ACE2) is an enzyme found in the outer surface cells such as nasal mucosa, nasopharynx, lung, stomach, small intestine, colon, skin, lymph nodes, thymus, bone marrow, spleen, liver, kidney, and brain. ACE2 regulates the renin-angiotensin system, which has an important role on regulation of blood pressure and heart functions. ${ }^{30,35-37}$

The genomic sequence result of (COVID-19) SARS-CoV-2 has shown 79.6\% identical with SARS-CoV-1 and $96 \%$ with bat coronavirus respectively. ${ }^{38}$ Furthermore, in vitro based recent experimental study using the ACE recombinant protein and ACE2 expressing cell lines indicated that the SARS-CoV-2 and SARS-CoV-1 showed the similar binding affinity to the host ACE2 receptor. ${ }^{18}$

S-protein has very essential role to produce a potentially effective antibody and vaccine. Recently the receptor-biding domain (RBM) in the S-protein apparently amino acid residue (Gln493) provide favorable interactions with human ACE2, which enabled the virus for human cell infections. This might be related the infectivity potential of the COVID-19. ${ }^{39}$ The amino acid sequence result analysis of SARS-CoV and SARS-CoV-2 SAR showed that similarity $75 \%$ in S-protein, $73.7 \%$ in receptor-biding domain (RBM) and $50 \%$ receptor binding motif $(\mathrm{RBM}) .{ }^{18}$

Since the COVID-19 (SARS-CoV-2) and SARS-CoV-1 showed sequence similarity, identify the common cross-reactive epitopes (CREs) is the key point. The shared CREs are similar epitope regions on the antigen surface among viruses that can be bound or neutralized by the same antibodies. Interestingly, very similar epitope was identified in between COVID-19 and SARS-CoV-1 at the biding site of S-protein with human ACE2 receptor. ${ }^{39}$ Recently, robust anti-ACE-2 Blocking antibody showed limited binding and neutralization activity against SARS-CoV-1. However, anti-ACE2 nonbanking SARS CoV-1 antibody showed positive cross-neutralizing activities to SARS-CoV-2. Indeed the reason is unknown yet. ${ }^{18}$ This might be due to the RBD conserved immunogenic part that antibody able to recognize in SARS-CoV-1 and the SARS-CoV-2. This provides a scientific evidence to develop a potent antibody or vaccine based on RBD against COVID-19.

Figure 2. ABO blood group. a phenotypic distribution of ABO blood group by race and Ethnicity, ${ }^{29}$ bRelationship between the ABO Blood Group and the COVID-19 Susceptibility. ${ }^{12}$

Histo-blood group antigens which are responsible for ABO blood group are highly expressed on epithelial cells and many other normal cells. ${ }^{40}$ In the same fashion, epithelial cells of respiratory and digestive tracts are the main site for replication of SARS-CoV-2 and SARS-CoV-1. ${ }^{6,37}$ Thus, SARS-CoV can produce the $\mathrm{ABH}$ carbohydrate epitope in the epithelia cells. S-protein of virions produced by either A or B individuals could be decorated with A or B carbohydrate epitopes, respectively. Naturally, with unknown reason we huma acquired natural anti-histo-blood group antibodies. These natural antibodies from blood group O, $\mathrm{B}$, and A individuals could bind to the S-protein and block its interaction with ACE2. Surprisingly, O blood group individual showed very low risk of infection by COVID-19 and SARS-CoV-1 as compared with non $\mathrm{O}$ blood group. ${ }^{12,28,41}$ The major reason is that the $\mathrm{O}$ blood group individuals have anti-A and anti-B antibodies in plasma. Interestingly, the plasma contained high anti-A antibody titer able to inhibit the S- 
protein interacting with ACE2 receptors. ${ }^{13}$ This indicated that ABO polymorphism has significant effect of the SARS-CoV outbreak further more study is needed to produce broadly neutralizing antibody and vaccine.

Green fluorescent tagged the C-terminal of S-protein was expressed in Chinese hamster ovary cells cotransfected with an $\alpha 1,2$-fucosyltransferase and an A-transferase (antigen A) in order to coexpress the Sglycoprotein ectodomain. The vitro result showed that anti-A monoclonal antibody and anti-A natural antibody from O blood group inhibited ACE2 and S-protein of SARS-CoV-1 interaction. ${ }^{13,42}$ Furthermore, convalescent plasma therapy taken from $\mathrm{O}$ blood group result in better recovery including for pregnant woman. ${ }^{22}$ In erectly, SARS-CoV-2 S- protein peptide are identified in human leukocyte antigen (HLA) complexes. The protein glycosylation has role in the adaptive immune response; this could leads for development of potential antibody and vaccine. ${ }^{43}$ Therefore, furthermore studies based on these evidence are suggested.

The potential of natural antibody in human varies from person to person. The natural antibodies possibly decrease over years in developed countries due to good environmental and person hygiene. Peoples who lived in less developed countries may elicit good natural antibodies. Plasma taken from O blood group with high anti-A antibody dramatically inhibits the interaction of S-protein with ACE2. However, Plasma from O blood group with low anti-A antibody did not inhibit S-protein and ACE2 in cell adhesion assay. ${ }^{13}$ ABO blood group based intervention of the COVID-19 may slowdown the fast spread the outbreak. Moreover, antigen-A based further study could lead to get potent antibody and vaccine.

Figure 3. SARS-CoV-2 entry to host cell and inhibition by antibody mechanism. The SARS$\mathrm{CoV}-2$ attaches by its spiked protein sub unit 1 (S1) to the host Angiotensin-converting enzyme 2 (ACE2) receptors. The host natural antibody and lab made and neutralizing monoclonal antibody can inhibited the interaction of the S-protein and host ACE2. The membrane fusion is carried out by its S2-proten. The RNA virus replicate inside the host genome and finally RNA virus released.

\section{Identify the immunogenic epitope and produce monoclonal antibody against COVID-19}

In recently years, monoclonal antibody play a tremendous role in therapeutic and diagnostics of many lifethreatening infectious disease. ${ }^{44}$ In the year of 2019, ZMapp antibody and the antiviral agent remdesivir were dropped out due to unsuccessful potential against Ebola virus. Later on, the monoclonal antibody MAb114 and REGN-EB3 showed more than $90 \%$ survivor rate of Ebola patients. ${ }^{45}$ It was also indicated that antibodies cocktail are more effective. ${ }^{46}$ Moreover, ZIKV-195, a potently neutralizing human monoclonal antibody, has shown significant therapeutic activity against Zika virus infection in a mouse model. ${ }^{47}$

$79.6 \%$ genomic sequence similarity and having the same human ACE2 receptor cells of SARS-CoV-1 and SARS-CoV-2 are the big scientific evidence opportunities. The potent neutralizing monoclonal antibody (CR3022, CR3014, m396, S230.15, R302, shown significant role in neutralization against SARS-CoV-1. ${ }^{48-51}$

Table 2. Neutralizing monoclonal antibodies against SRAS-CoV-1 and their sensitive amino acid fragments

\begin{tabular}{ll}
\hline Antibody name & Specific terminal, binding to specific amino acid domain, biding at S1-protein SARS-CoV-1 RBD \\
\hline CR3022 & C-Terminal RBD, 318-510 amino acid residue, Showed cross reactivity with SARS-CoV-2 \\
F26G18 & C-Terminal of RBD, 460-476 amino acid residues \\
4D4 & N-Terminal of RBD, 12-261 amino acid residues, inhibiting the post-interaction in the viral penetration i \\
CR3014 & C-terminal of RBD, 318-510 amino acid residues and 565 amino acid residue (high affinity) but do not sh \\
68 & 130-150 of the amino acid residues \\
80R & C-Terminal of RBD, 426-492 amino acid residues, blocking the interaction of S1 subunit protein with cell \\
F26G19 & C-Terminal of RBD, 359-362, 391-392, 424-427, and 486-492 amino acid residues \\
201 & C-Terminal of RBD, 490-510 amino acid residues, \\
1A9 & Binding to the Heptad repeat (HR) loops including heptad repeat 1 (HR1) and heptad repeat 1 (HR2) d \\
S230 & Binding to epitopes partially overlapping with receptor binding motifs on B domain of SARS-CoV-1, Blo \\
m396 & C-Terminal, 482-491 amino acid residues \\
\hline
\end{tabular}


In novel potent antibody and vaccine development, identifying the most sensitive amino acid fragment of the target antigen is very critical issue. Taking the advantage for the new study similarity in nucleic acid sequence and host ACE2 receptors in SARS-CoV-1 and SARS-CoV-2 is important. An impressive study was reported that S1 subunit protein amino acid residue from 318-510 bounds ACE2 host very efficiently than the full length S1 daman (amino acid residue 12-672). ${ }^{60}$ Furthermore, amino acid residue 327-510 and 318-490 did not detectably bind to detect ACE2. Interestingly, the major reason was investigated as the reason that about 7 amino acid cysteine which are 5 of them essential to ACE2 expression and the aspartic acid, glutamic acid amino acid which also have an important association with ACE2 are found within 318-510 amino acid (aa) residue domains. ${ }^{60}$

Figure 4. S1 subunit spiked protein SARS-CoV-1 amino acid residue domain. The aa represents for: amino acid residue, N: N- Terminal, C: C-Terminal.

In the same fashion, recently the receptor-biding domain (RBM) in the S-protein COVID-19 apparently amino acid residue (Gln493) showed favorable interactions with human ACE2 and the binding sites of S protein COVID-19 to human ACE2 were identified as residues 455, 486, 493, 501, and $505 .{ }^{39}$ So that, taking those critical scientific evidence could lead to produce robust neutralizing antibody and vaccine against COVID-19.

Surprisingly, from the recent study reported that, the monoclonal antibody CR3022 only showed a cross reactivity of SARS-Cov-1 with spiked protein of COVID-19. However, m396 and CR3014 failed to bind the RBD of COVID-19. ${ }^{48}$ This CR3022 is developed from convalescent serum SARS-CoV-1 patient B lymphocyte single chain variable antibody fragment $(\mathrm{scFv})$ phage display library. ${ }^{50}$ This antibody is targeted a C-terminal of sensitive amino acid residue from 318-510 of spiked protein RBD SARA-CoV-1 Moreover, this antibody is targeted spiked protein of RBD. ${ }^{48}$ Similar study was reported only ACE2-non-blocking SARSCoV-1 RBD antibodies was shown a positive cross reactivity with COVID-19. ${ }^{18}$ Thus, we suggest that further more studies and CR3022 may have the therapeutic and diagnostic potential alone or in combination with other neutralizing antibodies, for the prevention and treatment of COVID-19 infections. Production of novel antibody and vaccine based on those very important scientific backgrounds is an urgent needed. ${ }^{48}$

Plasma serum containing polyclonal antibody from fully recovered patient is as an immediate optional therapy against COVID-19 to slowdown spread and save live. ${ }^{24}$ However, getting enough recovered volunteer donors and transfusion compatibility remind as big challenges. In order to overcome this draw back, improved method for EBV (Epstein-Barr virus) immortalization transformation of human B cells which secrets a neutralization antibody have been developed. ${ }^{61,62}$ interestingly, specific monoclonal antibody isolated from EBV transformed B cell of patients recovered from severe acute respiratory syndrome coronavirus (SARS$\mathrm{CoV}-1$ ) infection resulted in vitro neutralizing efficiency from $10-8 \mathrm{M}$ to $10-11 \mathrm{M} .{ }^{63}$ Surprisingly, monoclonal antibody CR3022 which was developed from B cell SARS-CoV-1 recovered patients showed a dramatic cross reactivity binding potential for COVID-19. ${ }^{48}$ Blood group O individuals was shown very low risk of infection against COVID-19. Moreover, convalescent plasma taking from O blood group recovered individuals results better efficacy. ${ }^{12,13,22}$ Taking all together, isolation of specific monoclonal antibody from EBV transformed $\mathrm{B}$ lymphocyte of fully recover $\mathrm{ABO}$ blood group typically $\mathrm{O}$ could be an effective approach at this very critical emergency time to overcome COVID-19 pandemic.

\section{Conclusion and future perspectives}

In 21st century, COVID-19 is the most contagious pathogen and challangeful pandemic worldwide. Vaccine promote our body to produce antibody against targeted antigens most are helpful for preventive than treatment. By April 2020, there is no licensed drug or vaccine against COVI-19. Globally, more than 3 billion peoples are under quatrain, unemployment rate is increasing and consequences world economic crises. Very urgent vaccine and antibody development against COVID-19 is necessary. We summarized our manuscript in the following key points. 1. In order to slow down the fast spread, use a convalescent plasma therapy as an immediate therapy from fully recovered COVID-19 patents.

2. O blood group individuals are not susceptible against COVID-19 as compare non-O blood group. Con- 
valescent plasma therapy taken from $\mathrm{O}$ recovered individuals was shown good result for vulnerable people eventually for risky pregnant woman. Hence, ABO blood group genes involvement in related to COVID-19 outbreak further more study is encouraged. 3. Potent neutralizing monoclonal antibody can be isolated from EBV transformed immortal B cells of fully recovered COVID-19 O blood group patient. 4. Histo-blood group antigens which are responsible for ABO blood group and COVID-19 are highly expressed on epithelial cells of respiratory tracts. anti-A antibody containing plasma from $\mathrm{O}$ blood and lab produced anti -A monoclonal antibody inhibited the interaction of SARS Co-V S-protein and ACE2 host receptor.so that, further study based on this may have effective results for COVID-19 vaccine design and antibody production.5. Identify the efficient RBD of spiked glycoprotein of COVID-19. The domain which contains 318-510 amino acid residues have targeted amino acid residues to express ACE2 and RBD binding affinity hence, this domain based urgent vaccine and antibody development is important.

COVID-19 lack the necessary machinery to self-replicate and it dependent on the host cell machinery for propagation. Taking drastic prevention and control methods by the individual community helps to slow down the rapid COVID-19 pandemic and create a window time for the scientific researchers to develop effective vaccine and antibody as long-term goal is necessary.

\section{Acknowledgments}

This work was supported by National Nature Science Foundation of China (Grant No. 81870135), Construction Project of Fujian Medical Center of Hematology (Min201704), Special Fund from Fujian Provincial Department of Finance (2016B041), the Cooperation Project of University and Industry in Fujian Province (2017Y4005), Joint Research Project of Health and Education (WKJ2016-2-06), Joint Funds for the innovation of Science and Technology, Fujian Province (2016Y9032).

\section{Authors Contribution}

SKY and JH wrote the manuscript. JH revised and approved the manuscript. ML contributed in revising the manuscript. All authors have read and approved the final manuscript.

\section{Conflict-of-interest disclosure}

The authors declare no conflicts of interest.

Correspondence: Jianda Hu, Fujian Institute of Hematology, Fujian Provincial Key Laboratory of Hematology, Fujian Medical University Union Hospital, 29 Xinquan Road P.O.BOX 350001 Fuzhou, Fujian, China; Tel: +0086-13559353407; E-mail: jack123china@gmail.com

\section{References}

1. Chen N, Zhou M, Dong X, et al. Epidemiological and clinical characteristics of 99 cases of 2019 novel coronavirus pneumonia in Wuhan, China: a descriptive study. 2020;395:507-13.

2. Zhang Y. Initial genome release of novel coronavirus. 2020.

3. Wang L-s, Wang Y-r, Ye D-w, Liu Q-qJIJoAA. A review of the 2019 Novel Coronavirus (COVID-19) based on current evidence. 2020:105948.

4. Chen Y, Liu Q, Guo D. Emerging coronaviruses: Genome structure, replication, and pathogenesis. 2020;92:418-23.

5. Wang L-s, Wang Y-r, Ye D-w, Liu Q-q. A review of the 2019 Novel Coronavirus (COVID-19) based on current evidence. International Journal of Antimicrobial Agents 2020:105948.

6. Lu R, Zhao X, Li J, et al. Genomic characterisation and epidemiology of 2019 novel coronavirus: implications for virus origins and receptor binding. The Lancet 2020;395:565-74.

7. Lau SK, Woo PC, Li KS, et al. Severe acute respiratory syndrome coronavirus-like virus in Chinese horseshoe bats. 2005;102:14040-5. 
8. Liu Y, Gayle AA, Wilder-Smith A, Rocklöv JJJotm. The reproductive number of COVID-19 is higher compared to SARS coronavirus. 2020.

9. Zhang J-S, Chen J-T, Liu Y-X, et al. A serological survey on neutralizing antibody titer of SARS convalescent sera. 2005;77:147-50.

10. Shanmugaraj B, Siriwattananon K, Wangkanont K, Phoolcharoen WJAPJAI. Perspectives on monoclonal antibody therapy as potential therapeutic intervention for Coronavirus disease-19 (COVID-19). 2020:10-8.

11. Roberts A, Thomas WD, Guarner J, et al. Therapy with a severe acute respiratory syndrome-associated coronavirus-neutralizing human monoclonal antibody reduces disease severity and viral burden in golden Syrian Hamsters. 2006;193:685-92.

12. Zhao J, Yang Y, Huang H-P, et al. Relationship between the ABO Blood Group and the COVID-19 Susceptibility. 2020.

13. Guillon P, Clément M, Sébille V, et al. Inhibition of the interaction between the SARS-CoV spike protein and its cellular receptor by anti-histo-blood group antibodies. 2008;18:1085-93.

14. Sohrabi C, Alsafi Z, O'Neill N, et al. World Health Organization declares global emergency: A review of the 2019 novel coronavirus (COVID-19). International Journal of Surgery 2020;76:71-6.

15. Cucinotta D, Vanelli M. WHO Declares COVID-19 a Pandemic. Acta bio-medica : Atenei Parmensis 2020;91:157-60.

16. Winau F, Winau R. Emil von Behring and serum therapy. Microbes and Infection 2002;4:185-8.

17. Ahmed SF, Quadeer AA, McKay MR. Preliminary Identification of Potential Vaccine Targets for the COVID-19 Coronavirus (SARS-CoV-2) Based on SARS-CoV Immunological Studies. 2020;12:254.

18. Xie L, Sun C, Luo C, et al. SARS-CoV-2 and SARS-CoV spike-RBD structure and receptor binding comparison and potential implications on neutralizing antibody and vaccine development. 2020.

19. Hung IF, To KK, Lee C-K, et al. Convalescent plasma treatment reduced mortality in patients with severe pandemic influenza A (H1N1) 2009 virus infection. 2011;52:447-56.

20. Arabi Y, Balkhy H, Hajeer AH, et al. Feasibility, safety, clinical, and laboratory effects of convalescent plasma therapy for patients with Middle East respiratory syndrome coronavirus infection: a study protocol. $2015 ; 4: 1-8$.

21. Cheng Y, Wong R, Soo Y, et al. Use of convalescent plasma therapy in SARS patients in Hong Kong. $2005 ; 24: 44-6$.

22. Yeh K-M, Chiueh T-S, Siu L, et al. Experience of using convalescent plasma for severe acute respiratory syndrome among healthcare workers in a Taiwan hospital. 2005;56:919-22.

23. Bao L, Deng W, Gao H, et al. Reinfection could not occur in SARS-CoV-2 infected rhesus macaques. 2020 .

24. Liang T, Cai H, Chen YJTFAH, Zhejiang University School of Medicine. Compiled According to Clinical Experience. Handbook of COVID-19 prevention and treatment. 2020.

25. Mair-Jenkins J, Saavedra-Campos M, Baillie JK, et al. The effectiveness of convalescent plasma and hyperimmune immunoglobulin for the treatment of severe acute respiratory infections of viral etiology: a systematic review and exploratory meta-analysis. 2015;211:80-90.

26. Lloyd-Sherlock P, Ebrahim S, Geffen L, McKee M. Bearing the brunt of covid-19: older people in low and middle income countries. 2020;368:m1052. 
27. Batool Z, Durrani SH, Tariq SJJoAMCA. Association of ABO and Rh blood group types to hepatitis B, hepatitis C, HIV and Syphillis infection, a five year'experience in healthy blood donors in a tertiary care hospital. 2017;29:90-2.

28. Cheng Y, Cheng G, Chui C, et al. ABO blood group and susceptibility to severe acute respiratory syndrome. 2005;293:1447-51.

29. Dean L, Dean L. Blood groups and red cell antigens: NCBI Bethesda, Md, USA; 2005.

30. Walls AC, Park Y-J, Tortorici MA, Wall A, McGuire AT, Veesler D. Structure, Function, and Antigenicity of the SARS-CoV-2 Spike Glycoprotein. Cell 2020.

31. Li F. Structure, Function, and Evolution of Coronavirus Spike Proteins. Annu Rev Virol 2016;3:237-61.

32. Xiao X, Chakraborti S, Dimitrov AS, Gramatikoff K, Dimitrov DS. The SARS-CoV S glycoprotein: expression and functional characterization. Biochemical and Biophysical Research Communications 2003;312:1159-64.

33. Ou X, Liu Y, Lei X, et al. Characterization of spike glycoprotein of SARS-CoV-2 on virus entry and its immune cross-reactivity with SARS-CoV. 2020;11:1-12.

34. Li W, Moore MJ, Vasilieva N, et al. Angiotensin-converting enzyme 2 is a functional receptor for the SARS coronavirus. 2003;426:450-4.

35. Prabakaran P, Xiao X, Dimitrov DSJB, communications br. A model of the ACE2 structure and function as a SARS-CoV receptor. 2004;314:235-41.

36. Keidar S, Kaplan M, Gamliel-Lazarovich AJCr. ACE2 of the heart: from angiotensin I to angiotensin (1-7). 2007;73:463-9.

37. Hamming I, Timens W, Bulthuis M, et al. Tissue distribution of ACE2 protein, the functional receptor for SARS coronavirus. A first step in understanding SARS pathogenesis. 2004;203:631-7.

38. Zhou P, Yang X-L, Wang X-G, et al. A pneumonia outbreak associated with a new coronavirus of probable bat origin. 2020;579:270-3.

39. Qiu T, Mao T, Wang Y, et al. Identification of potential cross-protective epitope between a new type of coronavirus (2019-nCoV) and severe acute respiratory syndrome virus. 2020;47:115-7.

40. LE PENDU J, MARIONNEAU S, CAILLEAU-THOMAS A, ROCHER J, LE MOULLAC-VAIDYE B, CLÉMENT M. ABH and Lewis histo-blood group antigens in cancer. 2001;109:9-26.

41. Zietz M, Tatonetti NPJm. Testing the association between blood type and COVID-19 infection, intubation, and death. 2020.

42. Arendrup M, Hansen J-E, Clausen H, Nielsen C, Mathiesen LR, Nielsen JOJA. Antibody to histo-blood group A antigen neutralizes HIV produced by lymphocytes from blood group A donors but not from blood group B or O donors. 1991;5:441-4.

43. Grant OC, Montgomery D, Ito K, Woods RJJb. 3D Models of glycosylated SARS-CoV-2 spike protein suggest challenges and opportunities for vaccine development. 2020.

44. Waldmann T. Monoclonal antibodies in diagnosis and therapy. 1991;252:1657-62.

45. Mulangu S, Dodd LE, Davey Jr RT, et al. A randomized, controlled trial of Ebola virus disease therapeutics. 2019;381:2293-303.

46. Pettitt J, Zeitlin L, Kim DH, et al. Therapeutic intervention of Ebola virus infection in rhesus macaques with the MB-003 monoclonal antibody cocktail. 2013;5:199ra13-ra13. 
47. Long F, Doyle M, Fernandez E, et al. Structural basis of a potent human monoclonal antibody against Zika virus targeting a quaternary epitope. 2019;116:1591-6.

48. Tian X, Li C, Huang A, et al. Potent binding of 2019 novel coronavirus spike protein by a SARS coronavirus-specific human monoclonal antibody. 2020;9:382-5.

49. ter Meulen J, Bakker AB, van den Brink EN, et al. Human monoclonal antibody as prophylaxis for SARS coronavirus infection in ferrets. 2004;363:2139-41.

50. Ter Meulen J, Van Den Brink EN, Poon LL, et al. Human monoclonal antibody combination against SARS coronavirus: synergy and coverage of escape mutants. 2006;3.

51. Zhu Z, Chakraborti S, He Y, et al. Potent cross-reactive neutralization of SARS coronavirus isolates by human monoclonal antibodies. 2007;104:12123-8.

52. Berry JD, Hay K, Rini JM, et al. Neutralizing epitopes of the SARS-CoV S-protein cluster independent of repertoire, antigen structure or mAb technology. MAbs; 2010: Taylor \& Francis. p. 53-66.

53. Elshabrawy HA, Coughlin MM, Baker SC, Prabhakar BSJPo. Human monoclonal antibodies against highly conserved HR1 and HR2 domains of the SARS-CoV spike protein are more broadly neutralizing. $2012 ; 7$.

54. Greenough TC, Babcock GJ, Roberts A, et al. Development and Characterization of a Severe Acute Respiratory Syndrome-Associated Coronavirus - Neutralizing Human Monoclonal Antibody That Provides Effective Immunoprophylaxis in Mice. 2005;191:507-14.

55. Sui J, Li W, Roberts A, et al. Evaluation of human monoclonal antibody 80R for immunoprophylaxis of severe acute respiratory syndrome by an animal study, epitope mapping, and analysis of spike variants. 2005;79:5900-6.

56. Coughlin MM, Prabhakar BSJRimv. Neutralizing human monoclonal antibodies to severe acute respiratory syndrome coronavirus: target, mechanism of action, and therapeutic potential. 2012;22:2-17.

57. Ng O-W, Keng C-T, Leung CS-W, Peiris JM, Poon LLM, Tan Y-JJPo. Substitution at aspartic acid 1128 in the SARS coronavirus spike glycoprotein mediates escape from a S2 domain-targeting neutralizing monoclonal antibody. 2014;9.

58. Lip K-M, Shen S, Yang X, et al. Monoclonal antibodies targeting the HR2 domain and the region immediately upstream of the HR2 of the S protein neutralize in vitro infection of severe acute respiratory syndrome coronavirus. 2006;80:941-50.

59. Walls AC, Xiong X, Park Y-J, et al. Unexpected receptor functional mimicry elucidates activation of coronavirus fusion. 2019;176:1026-39. e15.

60. Wong SK, Li W, Moore MJ, Choe H, Farzan MJJoBC. A 193-amino acid fragment of the SARS coronavirus S protein efficiently binds angiotensin-converting enzyme 2. 2004;279:3197-201.

61. Lanzavecchia A, Corti D, Sallusto F. Human monoclonal antibodies by immortalization of memory B cells. Current Opinion in Biotechnology 2007;18:523-8.

62. Kwakkenbos MJ, Diehl SA, Yasuda E, et al. Generation of stable monoclonal antibody-producing B cell receptor-positive human memory B cells by genetic programming. Nature Medicine 2010;16:123-8.

63. Traggiai E, Becker S, Subbarao K, et al. An efficient method to make human monoclonal antibodies from memory B cells: potent neutralization of SARS coronavirus. 2004;10:871-5. 


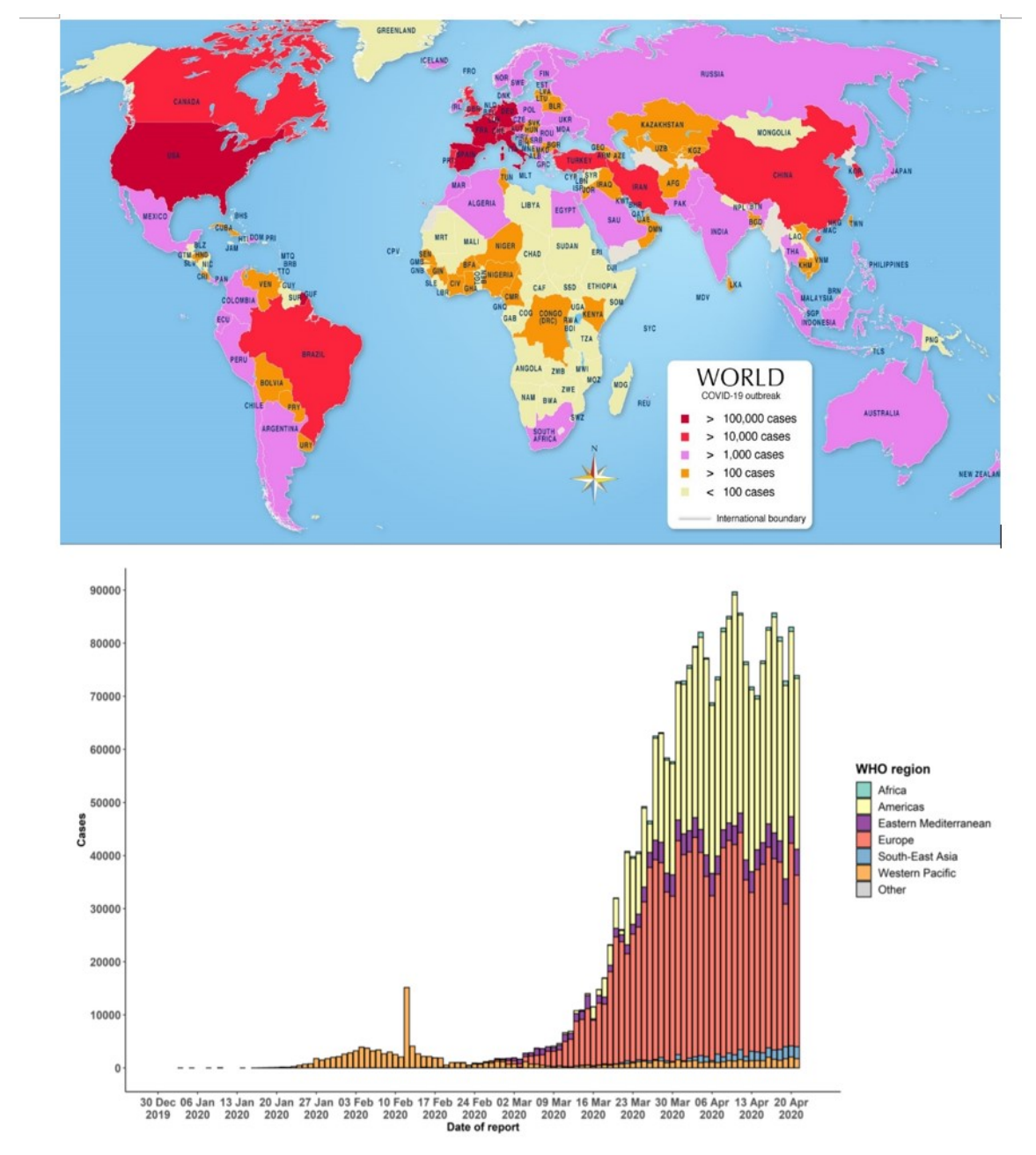


Distribution of $A B 0$ phenotypes by Race or Ethnicity

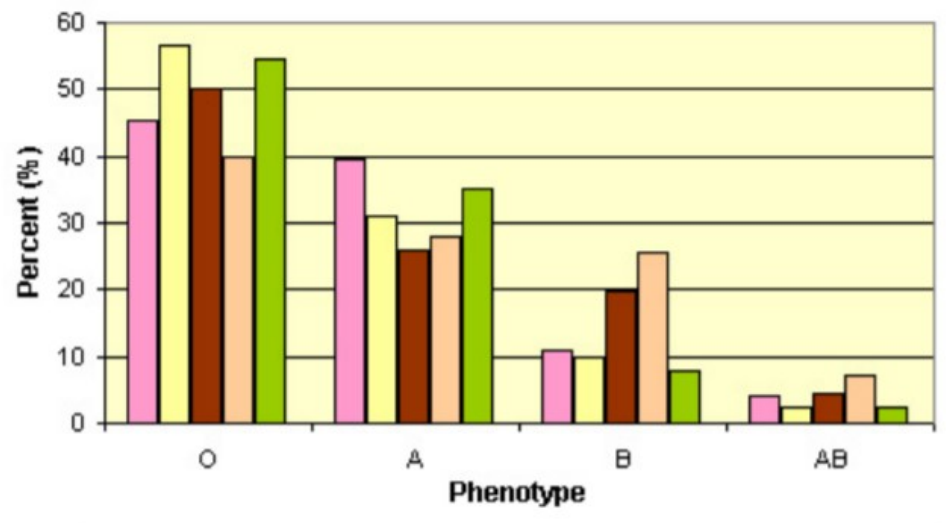

$\mathbf{A}$

White non-Hispanic

-Black non-Hispanic

$\square$ Hispanic

$\square$ North American Indian

口Asian

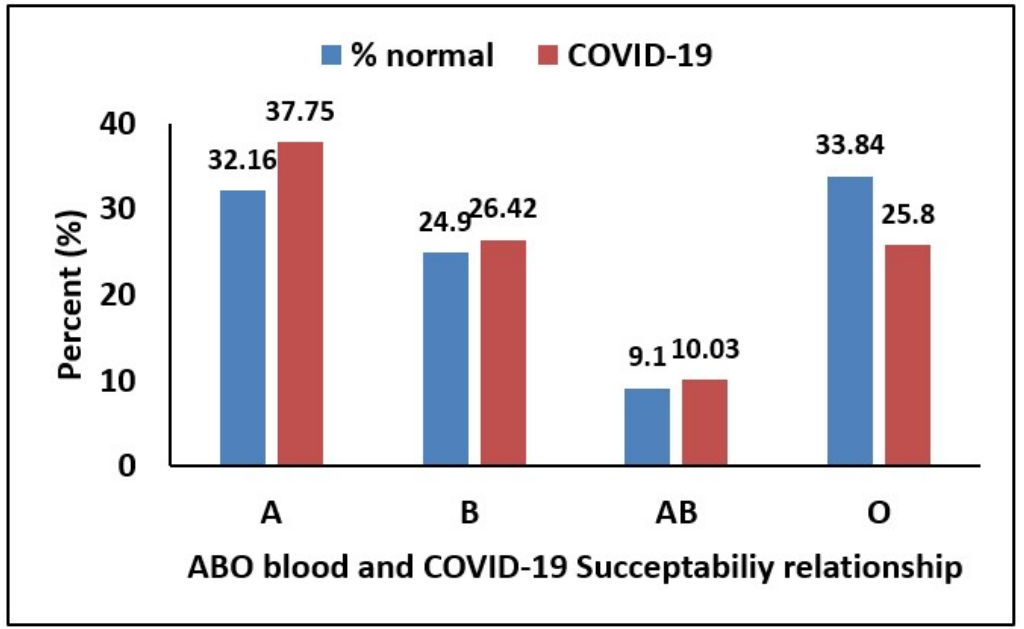

B 


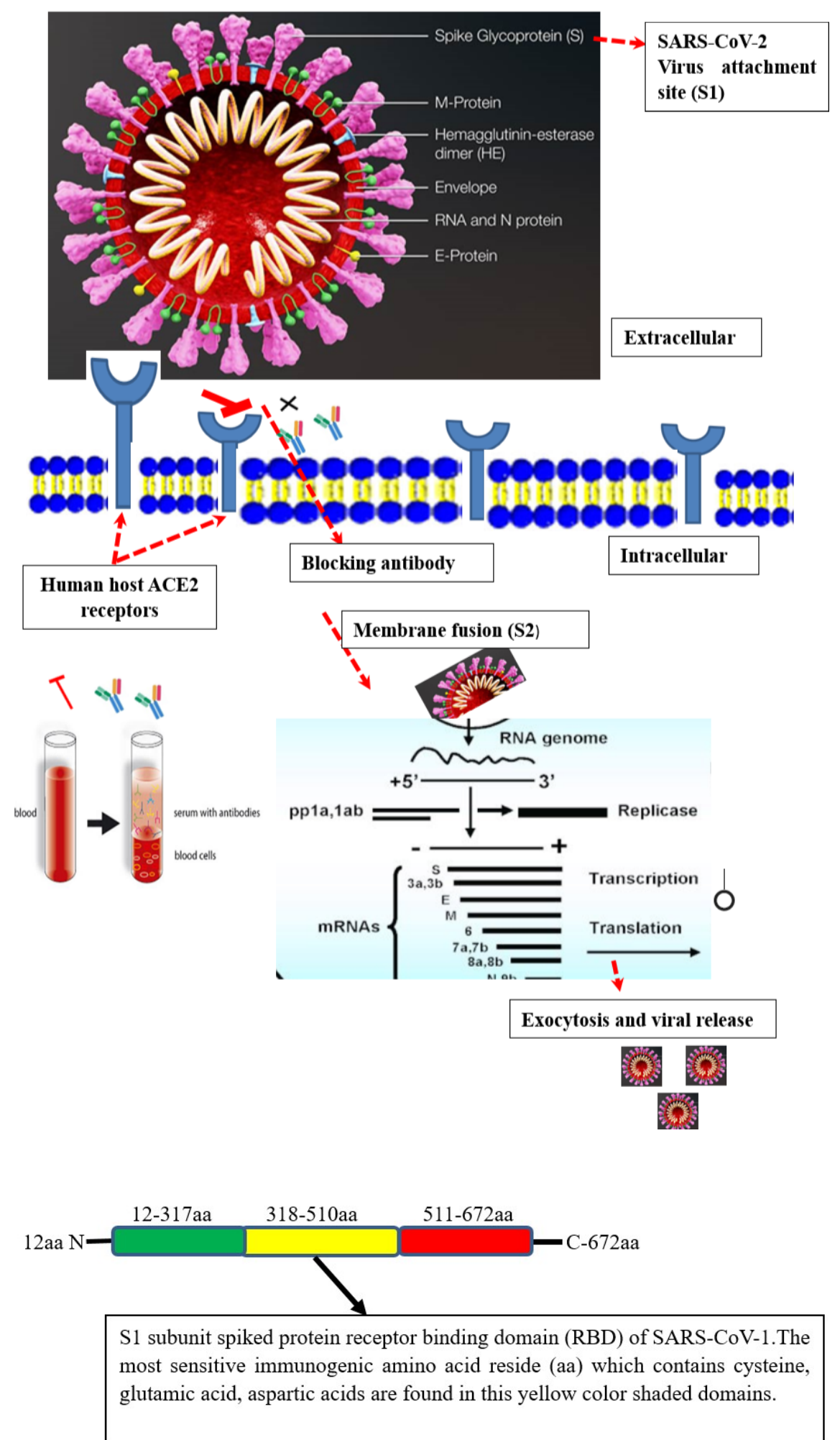

